\title{
Index of ailments and treatments
}

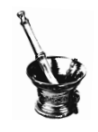

\section{page numbers in italics refer to illustrations}

Abbatis powder I70, I8I, I89, I9o, 222

abdominal constipation, see constipation

abdominal pain 2I, I63, I98, 219, 228-3o, 236

see also hypochondriac melancholy; stomach conditions

abdominal swelling, see inflammation and swelling acacia 83

'affection of the womb', see uterine conditions

agaric 79, 83, 92-3, 99-IOо, Iо9, II I, II4-I5, I I8, I23, I26, I35-6, I39, I48, I52, I58, I62, I74, І78-9, І83, І87, І90-- , І97, 200, 209, 218, 229, 235, 247, 261, 265, 267, 269, 277

agrimony $79^{-80}, 90, \mathrm{I} 53, \mathrm{I} 62, \mathrm{I} 73$, I79, I84, I90, I99, 204, 2067, 213, 218, 229, 233, 260

Agrippa's ointment I64

alabaster ointment I34, 22 I, 269 alephanginae 183 , I90-I, 200 alhandal (colocynth) 98, I57, I96, I99, 247 alkekengi, see winter cherry alkermes (confection) I03, IIO, I70-I, I79, I88-90, I92-4, I97, I99, 202, 207, 220, $242-3,254,25^{6}, 25^{8}-9,268$, 274,280

almond, see nuts aloe 8I, I23, I35, I38, I81, I84, I86, I9I, 2I $6,267,280$

rosata $\mathrm{I} 6 \mathrm{I}, \mathrm{I} 74$

alum 90, II5, I20, I37, I85

plume I55

rock $\mathrm{I} 33$

amber 83, 86, 88, 91, 95, гоо, го3, III, II3, I26, I35, I47, I49, І65, I66, I78, I87, I89, I9I, 206-7, 218-19, 228-9, 242

Crato's 79, 8I

fumes I 42,2 I I

spirit 267

white 140,207

see also oils

ambergris, see animal products ammoniac II9, I23, I7I, I73, I78, 201, 213, 230, 233, 276, 277

anal prolapse 42,88 angelica 84,85 , II5, II9 


\section{Index of ailments and treatments}

animal products

ambergris I6 I, I76, I97, 207

cantharides (beetle) I25

castor IO3, III, II3-I4, I3O, I7I, I83, I94, I97, 2I 7, 23I, 234-5, 244,267

cattle bile 159

chicken 258, 260

broth 94, I44, 273, 274

droppings I3I, 2 I9

fat I49, I99, 20I, 2II, 26o, 268 raw $23 \mathrm{I}$

civet $25^{8}$

crabs 94

dog droppings 2 I I

'droppings of fowls' I6 63

earthworms I69, I7I, 2 I3, 255, 260,265

fish glue 107

fox lung 166

frog spawn I35, I54, I92, 272

frogs 94, I93, 280

goat's kidney fat 180

goose droppings I38, I63, 2 I 3

goose fat 26o, 268

hartshorn 6o, 85, 98, I00-I, I03, I05, I08-9, II7-I9, I2I,

I3I-2, I4I, I44-5, I58, I63,

І65, I69, І76, I82-4, I87-9,

I92-4, 202-3, 208, 213,

$220-4,233,236,240-3,247$,

249-5 I, 254, 258-6o

hen feather 86

honey 62, 72, 79n., 93, II5-18, I27, I29, I33, I57, I59, I77, เ94, I96, г98-9, 203, 219, 26I, 290

rose 136, I64, I80, I9I, 201, 209, 212, 220

rosemary I40, I44

violet 94, 222

horse droppings 177

horse's hoof I57, 2I7, 244

ivory 85, I00, I03, II9, I2I, I24,

I53, I69, I79, I99, 243 lard 247

leeches 6o, Iо6, I24, I74, 207, $24 \mathrm{I}-2,273$

musk Iі6, І27, I32, I58, I87, I94, I97, 217, 24I, $25^{8}$

musk galls $2 \mathrm{I} 7$

mutton broth 155

ox gall i84

partridge 254, 260

peacock droppings 157

pigeon 38,240

pike's jaw I77

pork fat I42, I5I, I64

rooster 192

rooster gullet 89

scorpions, see oils

sheep suet 238

snails $85,94,193,243,260,280$

spiders' webs I3 I, 22 I, 224

stag's heart bone i io

swallows' nest 66, 2 I I

veal 94, I7I, I92, 233, 238, 254

wax 62, 84, 92, іло, II5, Iі9,

I22-3, I25, I32, I49, I70-I,

I77, I80-I, I89, 20I, 2I5, 238, 260

see also eggs; milk; human

products; oils

anise and aniseed 8o, 84, 9o, 99-IOO, IO4, IO9-IO, II4, II9, I 2 I- $3, \mathrm{I} 26, \mathrm{I} 32, \mathrm{I} 36, \mathrm{I} 42, \mathrm{I} 46$, I55, I58, I62, I65, I67, I76, I87, I90, І96, I98, 202, 207, 209, 2I2, 218, 260-I, 264, 269,272

see also oils

antimony 72,187

see also 'chymical cup'

appetite, see loss of appetite

apoplexy $\mathrm{i} 78$

apple 62, 81, 92, I00, I95, 264, 269, $273,274,276,278$

aquae (waters)

aqua vitae 94, II I, II3-I4, I64

aqua caelestis I5O, 224 


\section{Index of ailments and treatments}

aqua frigida Saxoniae 243, 249

aqua Mercurialis (water of mercury) 88, I04, 219

archonticon I70, I79, I89-90, I94, I99, 229-30, 239-40, 256-7, 259, 262, 265, 267, 277

aristolochia IO3

arthritis 2I, 59, 62, 25I-5

arum I70, I79, I90, 258, 267

asarabacca root II9

ash II9, I99, 264

asparagus II4, I58, I90, 207

assa odorata, see benzoin (gum)

assafoetida (gum) I03, III, II3, I20-I, I83, I94, I97, 2I7, 235, 244,247

auditory ailments I26-7, I74-5, 269-70

Augsburg syrup 207

aureae (pills) 83, 98, I I I, II3, I20, I26, I48, I53, I57

back pain, see backache

backache 21, 92, I33, I38, I47, I55, I63-4, I67-8, I92-3, I 97-20I, 220-25, 228-30, $237^{-} 9,245^{-6}, 25^{-}-\mathrm{I}, 263^{-6}$,

balm 274 268-9;

balsam (Indian) I70

balsam sulphur 226

barberry 6o, 85, I82, 213, 222, 223, 233,249

barley 92, 94-5, го8, г34, І36, І80, 202, 209, 222, 23I, 246, 272

cream of 94, I86, 2 I4

flour 94, 234

sugar 85 , II5-I6, I27, 2II, 216, 246

water 99, I40, I77, 2I 4, 246, 272

wheat 98, I73, 203, 205, 246, 297

basilicon ointment 234

bastard tertian fever see fever

bayberry I00, I04, II5, I27, I67, I85, Ig6 bdellium (gum) I I4, I8I

bean flour 86

beans I70

bedwetting 89

beer 79-80, гоo, I46, I53, I68-70, I74, I90, 20I, 2I8-I9, 230, 239, 255, 257, 26o, 262-6, 276-7, 280

beet I04, I27, 231, 272

beetroot 127

benzoin (gum) I03, II4-I5, II8, I67

betony $79^{-80}, 83,90,9^{8}-9$, $135^{-6}$, I40, I48, I58, I74, I83, I86, I9O, I99-200, 202, 204, 206-7, 23I, 242-3, 247, 260, 264,267

bezoar stone I06, I63, I88, I93, I94, 2I $3,223-4,233,242,245$, 249, 276;

water 225

bilberry I2 I

bindweed I9o, I96, 262

birthwort I35, I86

black bile, see humours

blackcurrant I08, 22I

bladder I77, 203-4, 214, 296

bleeding from the mouth $5_{5} 6-7$, 288-9

blessed thistle 93, 98-9, г26, I44-6, I79, 218, 223-4, 260, 264, 270

blotches, see skin disorders

bole Armeniac 83, 86, I07, I21, I24, I28, I33, I5I, I56, I77, I80-I, 21 4,272

borage 79, 90, 92, 94, 97, 99, 103-4, Iо8-9, II9, I52, I58, 207, $23 \mathrm{O}-\mathrm{I}, 233,238,269,273^{-4}$, pl. 8

borax 88 , I20

bowel disorders г23, I52, I66, г69, I74-5, I88-9, I98, 213, 233, $237,248-50,256,273,289$, 293 


\section{Index of ailments and treatments}

bowel disorders (cont.)

see also colic; constipation;

diarrhoea; dysentery;

lyentery

box-tree, see oils

brain 84, 234, 273-4, 289, 290, 29I, 292, 293, 294

bread and breadcrumbs $85,92,94$, I53, I54-5, I70, 246

breathing difficulties, see respiratory complaints

brewer's yeast, see yeast

briar I80, I96, 204

brooklime 2I, 79-80, 90, I68, I69-70, I73, I79, I84, I99, 218, 233, 237, 238, 244, 255-6, 259-6I, 264, 276, 277

broom II9, I58, I99, 207, 274 see also butcher's broom

bryony I37, I70, I94-5, 235, 257, $25^{8}, 267$

bugloss 79, 89-90, 99, го3, Іо9, гі9, I22, I3I, I38, I70-2, I79, I89-90, I93, I97, I99, 207, 23I, 238, 239-40, 242, 265, 268, 274, 280, pl. 9

burning fever, see fever

'burning urine' 8o-2, I38-9, I46-7, 202-3, 208, 2I4-I6, 215

see also urinary complaints

butcher's broom 207

butter 84, I34, I37, 205, 220, 249-50, 268, 28I

see also May-butter

cadmium $2 \mathrm{I} 4$

caelestis water, see aquae (waters)

calamine stone II5, 2I 4

see also cadmium

calamint I96, 202

'calcanso' i I5

camphor 79, 8I, 86, 94, I23-5, I5I, I56, I62, 22 I, 245;

red 215 'canker on the leg' I5I-2

see also ulcers and ulceration

cantharides, see animal products

caper iा9, I23, I73, I84, I9o, I99, 264, 267, 274, pl. I0

see also oils

carabe (carob) I77

caranna 84, 93, I22, I3 I-2, I46, I55, I58, I94, 243, $25^{8}$

caraway seeds 99-I00, IO4, IO9-IO, I2 I, I23-4, I27, I36, I46, I55, І62, I76, I87, I96, 218, 264, 269,274

cardiac passion, see heart

carrot $\mathrm{I} 55,262$

caryocostinum 9o, I85, 247

cassia 62, 81, 89, I07, I32, I76, 222, 23I, 268, 272

castor (beaver musk), see animal products

catarrh 82, 92, 97-8, I39-40, I49, I52-3, I9I, 2I8-I9, 278-80, 289

cattle bile, see animal products

celandine 85, I26, I63, 213, 264

celery 99

centaury I23, I90, 26I

ceruse I20, I25

chalk I26, I33, I5 I

chamomile 88, 90-I00, I04, I50, I55, I70-I, I80, 202, 204, $233,262,268,277$

see also oils

charlock I26

chaste tree I07

chest complaints 62, 98-9, II5-16, I $32-3,228,245,270-2$

constriction I68-9

expectoration $97^{-8}$, II $5^{-1} 6$, 205

'heaviness of the chest' $92-3$ 'pricking pain' $28 \mathrm{I}-2$ see also respiratory complaints chicken, animal products chickpea 203 


\section{Index of ailments and treatments}

chicory 79, 87, 89, 99-100, 108-9, II 4 , II8, I20-2, I34, I52-3, I58, I62-3, I90, 203, 206-7, 209, 213, 219, 233, 238, 24I-2, 250, 26I

childbirth 62-3, IOI, I I4n., I33-4, I7In., I72-3, 219-20, 230, 234, 236, 248n., 258, 296 see also puerperal fever china root 84, I0o, III, I36, I40, I53, I79, I92, 243

see also oils

choking 2I, I39, I42-3, I64-5, I95, I98, 218, 226, 258-9 see also respiratory complaints choler, see humours; tertian fever 'chymical cup' 45, 72, 85, 87, 95, 98-9, го7, I38, I4I, I43, I48, I50, I62-3, I65, 201, 205, 208, 267-9, 28I

cinnamon 79-80, 85, 90, 93, гоo, I04, I09-IO, II3-I5, II8-I9, I23, I30, I32, I36, I39, I4I, I52-3, I59, I62, I66, I73-4, I76, I79, I87, I90, I95-7, I99, 207, 209, 216, 218, 235 , $242^{-}-3,247,258,260-5,274$, 280, pl. I5

citron ı08-9, г6г, І76, І8г, г86, г97, I99, 22I, 242-3, 267, 274, 280 civet, see animal products clay I35 cleavers (goosegrass) I4O clove 93, IOo, IIO, II3, II5, II9, I2I, I23, I32, I53-4, I6 г, I67, I76, I9I, I96, I99, 201, 238, 243, $247,258,262,264,267,274$ see also oils clove-gillyflower I58, I70, I89-9o, I97, I99, 239, 243, 268 cochiae (pills) 98, I I, I48, I57, I65, I83

coconut 199

colchicum I20, I36, I39, I46, I53, I54, I58, I86, 2I8, 269 'cold gems', see gems

cold 89, I I2, I98, 224, 236, 242, 281, 289

see also cold in the head cold in the head 269-70 colic 79, 87, 93, I55, I72-4, 28I-2, 289

coltsfoot $84-5,89,93$, I66, I98, 244 comfrey I8I, I86, 202, 2I5 comitissa ointment I07, I78 constipation $\mathrm{I} 72-4$, I98-200, 204-5, $233,237,255^{-7}$

constriction of the chest, see chest complaints

convulsions 2I, I8I-2, I98-200, 234-5;

arms $257-8$

eyes 2I6-I8, 234-5, 244-5

face $234^{-5}, 239^{-} 4 \mathrm{I}, 257^{-8}$

hands $257^{-8}$

jaw $167-8$

limbs I8I -2

see also epilepsy

copperas I5I

coral 79, І03, Іо6, І08, Іі7, І3г, І46, I89, I94, 2I6, 223, 233, 245, $25^{\mathrm{I}}$

red I08, Ііо, II7, І24, I76, I8I, I87, I99, 203, 272, 276

white 178

'cordial flowers, the' 84, I I9, I2I-3, I73, I90

coriander 8o, I00, I07, IIо, II5, II9, I21, I23, I36, I46, I62, I66, І76, I80, I87, I96, 218, 26o, 264, 269

\section{Roman I67}

costus II4

see also oils

couch-grass I9o

coughs and coughing $83-5,94-5$, I 5 -I8, I26-9, I32-3, I65-6, I98, 243, 245-6, 270, 278-8I, 288-9

see also respiratory complaints 


\section{Index of ailments and treatments}

cream, see milk

cream of tartar I39, I4I, I69, I76, I78-9, I82, I85, I90, I98, 227, 229-230, 242, 247, 25I, 256, $262,263,267,273,275^{-6,280}$

crocus 6o, 223, pl. I9

crocus Martis (iron oxide) 89, 92, I26, I52, I57

cubeb (berry) I04, I59

cucumber I96, 2I I, 23I

cumin I04, I27, I54-5, I67, I80, I86, 199

cure-all ${ }_{5}{ }^{8}$

currants 80, I63, 2 I 3

cyclamen I58, I64

cypress II 5

see also nuts

Cyprus turpentine, see turpentine

daisy I28, 2 I4, pl. 20

damask roses I70, I79, I9o, I99

see also roses

'darkened vision', see eyes and eyesight

dates I33, 254

deafness, see auditory ailments

diabetes ('dropsy of the chamber pots') 240, 289-90

diacatholicon 93, 99, I20, I30, I32, I50-I, I63-4, I69, 205, 213, $23 \mathrm{I}, 237,240,260,28 \mathrm{I}$

diacorallion I7 I

see also coral

diacrocuma 233, 256, 262, 276

diacubeb i6 I, 230

see also cubeb

diacyminum 158

diagrydium I26, 235, 265

diamargiton 274,280

diambra I7I, 20I, 242, 262-3, 267, 269

see also amber

diamoschum IIO, I7I, I9I, 207, 242, 263,269

see also musk dianthos 197

diaphoenicon 85, 93, 99, I05, II6-I7, I50, I63-4, I80, 202, 205, 213, 237, 250, 256, 28I

diarrhoea 64, 97, I52, г66-7, I74-6, I8I, I88-9, 289

diascordium 99, Іо8, I45-6, І5г, г76, I88, 24I, 245

diatartar I90, I9I, 264, 269, 276

see also tartar

diatragacanth I07, I27, 212, 246, 265,274

see also tragacanth

diaturbith powder $148,233,256$, 260-I

see also turbith

dill I7I, 26I

see also oils

dittany I7I

white 195,247

dock 99, I53

dodder I04, I09, II9, I99, 216, 269,

274

droppings and dung, see animal products

dragon's blood 83, 86, І24, І28, І78, I80

dropsy 99-Ioo, I39-40, I59-6г, I63-4, I78, I96-7, 229-30, 254, 258-63, 29I, 296

see also diabetes; 'dropsy of the chamber pots'; tympany

'dropsy of the chamber pots', see diabetes

'Dudley's powder' i87, 230

dwarf-elder igo

dysentery 64, 92, I52, I8o-I, 290

earthworms, see animal products

eggs 94, I70, I77, I86, 255

egg white $83,85-6$, I07, Io9, I24-6, I33, I38, I77, 2 I4, $22 \mathrm{I}$

soft-boiled 85, 89, I24, I28

'water of eggs' 2 I5 


\section{Index of ailments and treatments}

yolk 92, 94, II5, I26, I28-9, I77, I80, I98, 203

elder i i4, I70, I90, I96, 259, 26o

see also dwarf-elder; oils

elderflower 221, 262

elecampane 79, 84, 85, I I9, I2 I-3, I29, I32, I37-8, I58, I62, I70, I89-90, I96-9, 207, 267-9, pl. I6

emerald, see gems

endive 89, 203

epilepsy 6I, 63, IOI-3, IIO, I8I-3, 290

see also convulsions

eringo I99

euphorbia I86

'exhausted appetite', see loss of appetite

expectoration, see chest complaints

eyebright I24, 245

eyes and eyesight $77-8,81,83,86$, III-I3, I22-5, 200-I, 206-7, 2І6-I8, 234-5, 240, 244-5, 269-70, 295

see also flux; rheum

fainting and faintness $77,82,9 \mathrm{I}$, 98-9, І2 I-2, I57-8, I69, I88, I90-I, 248-9, 258-9, 266-7, 278

see also giddiness

fennel 79-80, Iо0, I04, IIо, I I4, II9, I 2 I- 3, I $27, \mathrm{I} 36-7, \mathrm{I} 54, \mathrm{I} 5^{8}$, І67, І76, І87, І90, І96, 202, 207, 209, 245, 259, 26I-2, $265,268,272,274$

see also oils

fenugreek I25, I80, 2I I, 234, 268 fern 204

see also hart's-tongue fern; horsetail fern; maidenhair fern

fever 25, 98-9, IOI, IO4-7, II5, II7, I3 ${ }^{\mathrm{I}-2}, \mathrm{I} 4 \mathrm{O}-\mathrm{I}, \mathrm{I} 8 \mathrm{I}-3,208$, 230, 244-5, 250-I, 263, 28I-2, 292, 294, 295 bastard tertian 59, 20I-2, 222-5, 288

burning 59-6o, I43-4, I63-5, I $83-4,204-5$, 208, 220-2, $275^{-7}, 232-4,239^{-4}$ I, 245-6, 258-6I, 275, 289

erratic IOI, II3-I4, I93, 275-8 290

hectic $94-5,292$

malignant 45, I43-4, I49-5 I, $275^{-7}, 293,296$

'new' I $43-4$

puerperal ('milk') I45-6, 230n., 236

quartan $23^{2-} 3,294$

quotidian $145^{-6}$, I93-4, 242-3, 295

scorbutic I93-4, 232-5, 248-50 tertian 85-6, 95-6, 99--IOI, I34, $222-5,232-4,288,295,296$

'Ungaric' 45, I07-9, 295, 296 see also 'Hungarian disease'

feverfew I5O, I55, 224, 257

figs I 5 , I34, I42, I65, 205, 212, 246, 272

figwort (scrophularia) 84

fish glue, see animal products

fistula 159

'fit of the mother', see uterine conditions

flowers of sulphur, see sulphur

flowers of yeast, see yeast

flux I27, 209, 239-40, 289, 29I-3, 296

eye $83,1^{2} 4^{-5}$

belly I80-I, I88-9

bloody 64, I80-I, I88-9, 204, I30-I, I33, I52, I55, 237

haemorrhoidal 239-40

menstrual I30, I33, I52, I55, 237

mouth I2O-I

red 87, I25-6, I30-I, I33, I52, I55, I92, 29I

uterine 98

white I07, I33, I77-8, I92-3, 228-30, 29I 


\section{Index of ailments and treatments}

foetidae, see assafoetida

fox lung, see animal products

frankincense 84, 86, I I5, I9I, 238

'French disease' 43, I47, I78

French lavender, see lavender

French wormwood, see wormwood

'frenzied hunger', see hunger

'frenzy after childbirth' 230-2

see also puerperal fever

frigida Saxoniae, see aquae (waters)

frogs and frog spawn, see animal products

fumitory 79, 90, I00, I09, I20, I22, I25, I29, I62, I73-4, I79, I90, I99, 203, 25I, 26o-I, 264, 267,276

galangal I04, IIO, II5, II9, I2I, I23, I39, I53-4, I96

galbanum (gum) I I4, 2 I7

Galen's laetificans, see laetificans

galls, see oak (galls)

gamboge (resin) I00, 262, 265, 277, 278

garlic 127, I37

garnet, see gems

gems I03, I08, 274

'cold gems' 99, 240

emerald I08, II7, 22I, 274

garnet I08, II7

ruby I08, II7

salt of gems i86

sapphire io8

gentian I23, I32, I90

germander 79, 93, I19, I22, 26o, $264,267,274,276$

giddiness 6I, I22-4, I57, I74-5, I90-I, 206-7, 228-30, 29I

ginger 92, 93, 99-100, I04, Io9, I Iо, II9, I2I, I23, I26, I4I, I54, I79, I86, I90, I95-7, I99, 235,268

goat, see animal products; milk gold I08, II7, I90, 218

see also litharge of gold golden-rod 204

gonorrhoea I46-7, I77-8, 2I4-I6, $215,29 \mathrm{I}$

good-King-Henry (goosefoot) 205 goose droppings, see animal products

gourd ii $6,222,23$ I

gout I54, I85-6, 246-7, 254, 29I-2 grains of paradise 104 gravel-root $\mathrm{I}_{3} 8$

green bile I43-4, 222

ground-ivy 84

ground-pine II9, I22, I86, 217-I8, 270,274

see also pine

guaiacum i6, 80, 84, 86, 90-гоo, I07, III, II4, I36, I46, I5I, I59, I66, I77, I79, 2I6, 262, 267,269

gum ammoniac II9, I23

gum Arabic I77

gums 21, I35-6, I52-3, I56, I67-8, I90-I, I98, 200-I, 237, 264, 295, 288-9

see also toothache

gypsum 83 , IO7

haematite I2I, I25, I80, 272

haemorrhoids II4-I5, I74, 225-6, 239-4I, 292

see also veins

hart's-tongue fern I73, I99

hartshorn, see animal products

hazel, see oils

hazelnut, see nuts

headache 6I, 95-8, I00, I09-10, I22, I30, I34, I36-7, I43-4, I46, I57-8, I6I, I74-5, I90-3, I98, 206-8, 216-I8, 220-5, 234, $242-3,263-6,268-9,294$

hearing, see auditory ailments heart I7, 6I, I08, II0, II6, I84, 22I, $234,24 \mathrm{I}, 274$

'cardiac passion' I98-200

pain ${ }^{3}{ }^{\circ}$ 


\section{Index of ailments and treatments}

palpitations I22-4, I7 I-2, 228-30, $236,244^{-5}, 248-50,267$, 269-70, 277-8I, 292

'pricking' IO9-IO

heartburn 2I, 9I, II8, I90-I, 268-9, 292

see also stomach conditions

hectic fever, see fever

hellebore 216, 250, 273

black I03-4, I09, I83

white $\mathrm{I} 34,243$

hemiplegia 258, 292

see also paralysis

henbane 127

hiccups 144

hiera 194

hiera picra I00, I99, 202, 257

hiera simplex 9I

hiera with agaric I78-9, I87, I90-I, I97, 200, 218, 229, $235,247,265,277$

hippocras 104

hoarseness II $5^{-16}$, I96-7

Holland powder 93, 99, I37, I57, 202, 237, 238, 256-7, 267, $275,28 \mathrm{I}$

'holy powder', see Holland powder honey, see animal products

hops I04, I38

horehound 84, 93, I38, I40, 2 I9

white 190

horse, see animal products

horse-tail fern $2 \mathrm{I} 4$

horseradish 264

houseleek 8I, I62, 24I

human products

'powdered unburied human skull' I83

'urine of a prepubescent boy' $26 \mathrm{I}$

see also milk

humours ${ }^{1} 5^{-1} 6,3^{8-40}, 84,91$, I26, I43, I96, 200, 207, 209, 231, 245n., 252, 254, 273-4, 289-97 black bile I5, 21, I43, 203, 257-8, $270,274,296$ see also melancholy

blood I5, I05, I06, 2I3, 227, 26I, 270, 288-9, 29I-4 see also bleeding from the mouth; flux; nosebleed; passing blood; spitting up blood; vomiting blood

choler I4I, I43-4, I78, 2 I3, 288-90, 292, 296

phlegm I5, 87-8, II5-I6, I40-I, I43-4, I98, 206-7, 220-2, 243, 257-6I, 274-6, 288, 29I, 295, 297

yellow bile 15,232

see also green bile

'Hungarian disease' I43

see also fever, 'Ungaric'

hunger $\mathrm{I}_{47}-8$

see also loss of appetite

hyacinth 242

hydromel i 5

hypochondriac melancholy 100-3, IO9-IO, I22-4, I49-5I, I90-I, 263-6, 269-70, 277-81, 292

see also stomach conditions

hyssop $84-5,89,93$, I03, I28, I46, I58, I64, 243, 259, 273, 28I

Indian balsam, see balsam (Indian) inflammation and swelling 289, 290, 29I, 293, 294, 296

abdomen I63-4, I72-4, 288

ankles I09-10, I85-6, 254-5

belly $196-7,26 \mathrm{I}-3$

eyes III, I22, 295

see also rheum

face 77,82, I20, I49, I59-6I

feet 89-90, 99-100, I54, I59-6r,

I93-4, 237, 254-5, 26I-3,

$276-7$

fingers $154,264-5$

gums I $35^{-6}$, 264-5 


\section{Index of ailments and treatments}

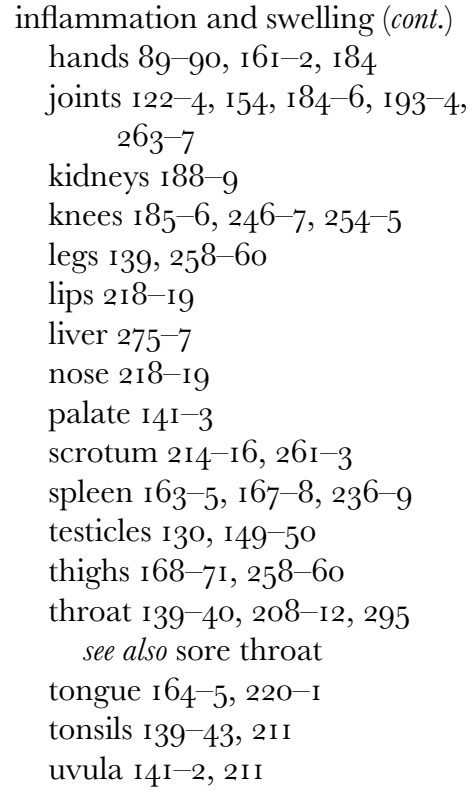

see also diarrhoea; dysentery; gout; haemorrhoids; melancholy; pleurisy

iris 89, II4, II5, I22, I29, 267

iron water $20 \mathrm{I}$

itches and itching 88, 90-I, I36-7, I6I-2, 24I, 247

ivory, see animal products

jaundice $\mathrm{I}^{8} \mathrm{I}-3$

splenic 178

yellow $85^{-6}$, I38, I62-4, I68-7I, 2I $2-\mathrm{I} 3,222-5,232-4,26 \mathrm{I}-6$, $270-2,292$

jujube 98,203

juniper 127

berries 80, I68, I70, I99, 218, 260, 264

seeds I7I

wood 84, II9, I29, 274

kidneys 92, I77, I88-9, I9I-2, 203-4,

$$
\text { 290, 294-6 }
$$

see also stone

knapweed (herb trinity) 84 knee I68-7I, I85, 246, 254

see also inflammation and swelling

labdanum 84, 93, I I0, I28, I32, I49, I89, 20I, 24I

lady's mantle 204

laetificans I03, IIо, I76, I87, I89, I93-4, I97, 274, 280

larch II 5

lard, see animal products

laudanum I22, I44, I52, I94, 202, 238,269

laurel 87,90 see also oils

lavender IOO, II4, I24, 207, 267

French II4, I66-7, I79, I83

lead I5O, 2I4-I5, $23^{8}$

red $149,215,25^{8}$

sheet I77, I92, 203, 2I4-I5, 2 I9

white $\mathrm{I} 5 \mathrm{I}$

leeches, see animal products

lemon 98, IOI, I05, IO9, I2O, I40-I, I5I, I76, I84, I93, 216, 223-5, 240, 258-9

lemon balm 88, ir9

lemongrass $\mathrm{I} 73$

leprosy 88

lettuce 94, 22 I, 23I

lilies 89

white I7I

see also oils; water-lilies

linctus, see lohoch

linseed I25, I30, I80, 2I I, 234, 268

see also oils

liquorice 8I, 84-5, 98, I08-I0, I I4, II 6 , ІІ9, І2 I, І23, І28-9, І32, I34, I36, I42, I46, I53, I58, I62, I64-6, I73, I77, I79, I85-6, I90, I98-9, 202-3, 205, 207, 209, 2II-I2, 2I4, 218, 244, 246, 259-62, 264, 269, 28I, pl. 5

litharge of gold 120

liver 22-4, 9I, I39, I53, 229, 232, $234,275,292-3$ 


\section{Index of ailments and treatments}

lohoch (linctus) 62, 85 89, I29, I64, I66, I98, 205, 212, 243, 258-9

sanum et expertum $85, \mathrm{I} 66$

London treacle 87, 9I, 237-8, 268

long pepper 98, I04, I26

see also oils

loose bowels, see diarrhoea

loss of appetite 6 I, 82, I4I-2, I50-3, I65-6, I68-7I, I78-9, 204-5, 206-7, 278-9, 292

see also heartburn

loss of movement, see paralysis

loss of speech $234^{-5}$

lovage I55

lungs II $5^{-\mathrm{I}} 6,226-7,245^{-6}, 27 \mathrm{O}^{-2}$, 288-9, 295

ruptured vein I2 I

see also respiratory complaints

lyentery 64, I8I, 293

see also diarrhoea; dysentery

mace 80, 97, II5, II9, I24, I39, I4I, I67, I96, I99, 238

see also oils

Macedonian parsley 247

see also parsley

madder I I4, I90, I99

magisterium of pearls I46, I89, I94, 202, 223, 243

maidenhair fern 84, 85, II7, I2I, I73, 207, 212, 243, 246, 272, 28I, pl. I8

malignant fever, see fever

mallow 81, 94, I04, I54, I77, I83, I99, 202, 205, 214, 231, 237, 259, 268, 272

see also marsh mallow

manna 62, I00, I09, II7, I30, I32, I49, 206, $25^{\mathrm{O}}$

manus Christi (cordial) 85, I88, I92-4, 233, 240, 280

marigold 6o, I3I, I99, 213, 223, 237 marjoram II5, II9, I2I-4, I27, I67, 236 marsh cinquefoil I70

marsh mallow 8I, 84, I34, I37, I38, I49, I7I, I86, I99, 202, 204-5, 2II, 222, 237, 260, 268, 272, 276, 28I, pl. I7

'Martiatum ointment' 90, III, II3, I58, I64, 257

mastic 84, 86, 89, I I5, I23-5, І28-9, I32, I35, I6I, I74, I76, I78, I80, I86, I9I, 216, 265

see also oils

May-butter I37

measles I5, 6I, 245-6, 275

mechoachan 99-I00, I09, II4, I53, I6I, I86, I90, 265

melancholy I0, 21, 40-I, 4I, 63-4, 70-I, IOO-I, IO4-7, IO9, II8-I9, I22, I49-51, I72-4, I83n., I93-4, I97-200, 22830, 236-7, 248-50, 253-4, 266, 269-70, 272-5, 277-80, 290, 292-5

see also black bile; hypochondriac melancholy

melilot I49-50, I78, I80, 202, 223, 268, 277

melon 95, 2II, 272

see also water-melon

menstruation $\mathrm{I} 5,20,89,94^{-5}$, II I-I2, I25-8, I30-I, I33, I39-40, I44, I52, I55, I58, I62-3, I7I-4, I78, I82, I95, 216-I8, 228-30, 233, 237-8, $244-5,272-5,294,296$

see also flux mercurius vitae Iı6, II7, I47, I48 mercury (plant) I04, 237, 272 mercury water, see aquae (waters) milk 62, 93-4, I24-5, I3 ${ }^{\mathrm{I}-2}$, I37-8, I40-I, I43, I5 I, I86, 2I $4^{-1}$, 219, 225, 236-7, 247, 260;

cow's 8ı, 85, I38, I70, 245

cream 85

curd $\mathrm{I}_{4} \mathrm{O}-\mathrm{I}$

goat's I2I, I70, 245 


\section{Index of ailments and treatments}

milk (cont.)

human 83,94

steeled 177

whey 105, I26, I44, I92, I95, 235, 237,272

milk fever, see puerperal fever

millet Ioo, I67

mint 88, 97, I00, I03

miscarriage $98-9$, I27-8, I34

Mithridate 87, 9I, I3I, I45, I52, I58, I $7 \mathrm{I}-2, \mathrm{I} 82-3, \mathrm{I} 88-9,234$

morphew I24, 264

see also skin disorders

motherwort I23, I26

'motion of the womb', see uterine conditions

mouth, see spasm of the mouth

mugwort I30, I40, I58, I72, I90, I95, I97, 204, 23I, 235

mulberry I36, I40, I44, 20I, 209,

mullein 84 $212,220,246$

see also oils

mummy 103, I77

musk, see animal products

musk galls see animal products

mustard, II $5^{-1} 6$

mutton broth, see animal products

myrobalans I09, I86

myrrh 86, II3, II6, I23, I25, I30,

myrtle I28, I77 I32, I66, I74, I8I, I84, 234

see also oils

nard, see oils

naval pitch I03, I28

nephritic plaster 238

see also kidneys

nettles 93, I04, I55, I58, 224, 256, 259, 264

'new fever', see fever

nigella II5, II9

nightshade I25, 238, pl. 6

nocturnal emission, see seminal discharge nosebleed I35, 220-2, 293-4

nuts

almond 94, II6, 246

cypress 128 , 180

hazelnut I 3 I

pine II 6

pistachio IIO

walnut 22I, 224n.

see also oils

nutmeg 20, IOO-I, IO3, IIO, II 3, II5, II9, I2I, I23-4, I54, I58, I76,

see also oils I79, I83, I87, 258, 264

oak

bark 155

galls $83, \mathrm{I} 24, \mathrm{I} 28, \mathrm{I} 80$

leaves 90,137

moss $\mathrm{I} 28, \mathrm{I} 78, \mathrm{I} 83$

see also polypody of the oak oils

almond 6r, 84, I09, II5, I34, I49, I99, 205, 22I, 250, 268, 28I

amber II3, I84, 257, 267

anise Ioo, I98, 263, 265, 269

box-tree $225^{-6}$

capers II9

castor 217,260

chamomile I49, I50, I7I, 2I I, 260

china 200, 207

cloves I23

costus I I3

dill I I9, I99

elder 225

fennel 265

hazel wood 2I2, 24I;

laurel III, II3

lilies 2I I, 2 I7

linseed I04, I30, I50, I7I, 225-6, $25 \mathrm{I}$

mace 93, IIO, I22, I32, 201

mastic 201

mullein I7o

myrtle I78, I80

nard II9, I23, I64 


\section{Index of ailments and treatments}

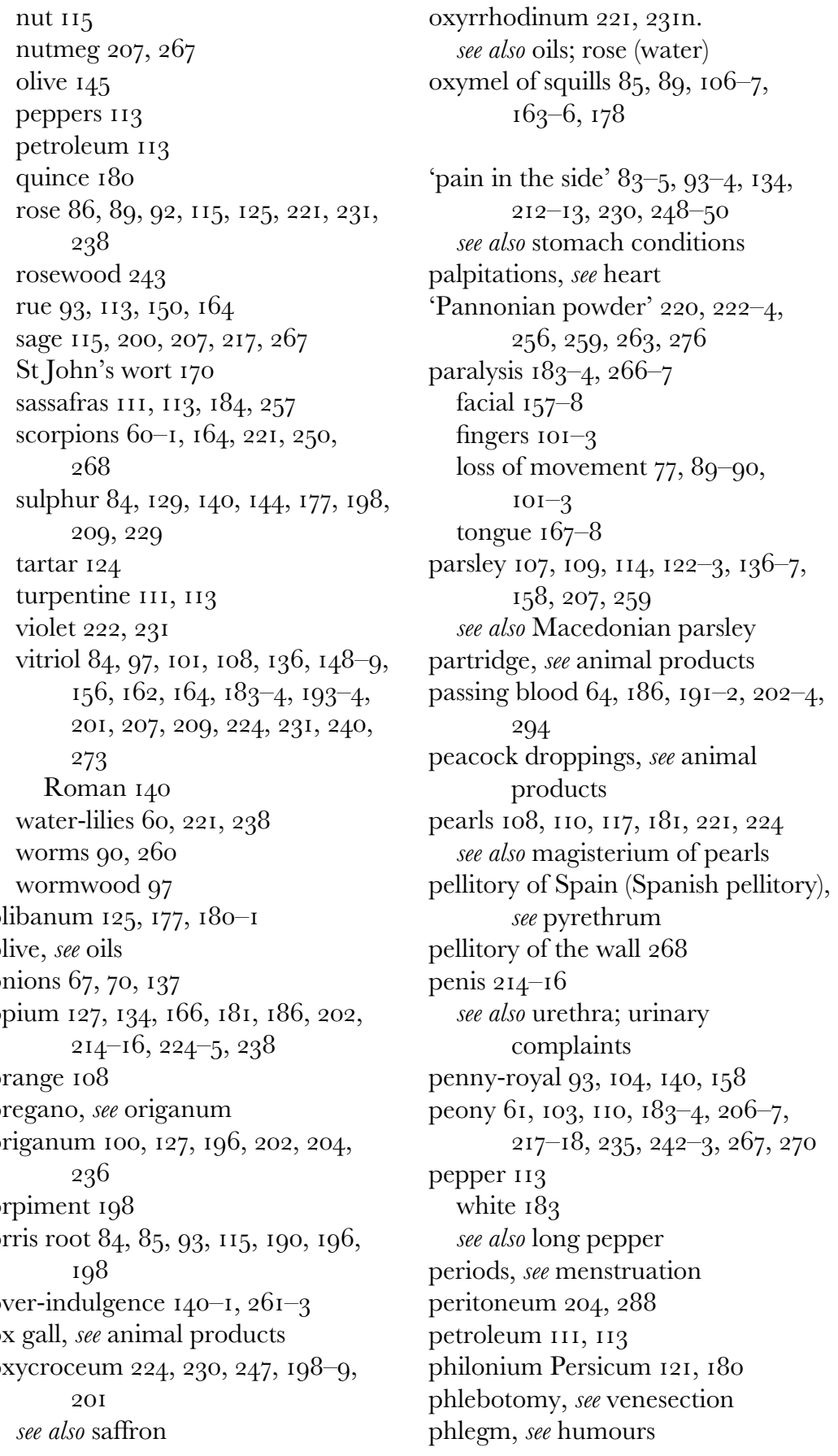




\section{Index of ailments and treatments}

phthisis I03-4, 294

pigeon, see animal products

pike's jaw, see animal products

pimples, see skin disorders

pine leaves II 4

see also ground-pine; nuts

pistachio, see nuts

placenta ('retained and corrupted products of conception') 234

plantain I07, I20, I2I, I25, I46, I64 I80, I9I, 203, 209, 212, 2I4, 2I6, 22I, 23I, 245, 272

pleres archonticon, see archonticon pleurisy 2IO, 28I-2, 294

plume alum, see alum

plums 272

polypydy of the oak (fern) I04, Io9, I36, I53, I58, I62, I73, I85, I99, 2I8, 26I, 269, pl. 2 I

pomegranate 6 I, 83, I08, I25, I28, 2I 2,2 I $4,22 \mathrm{O}^{-} \mathrm{I}, 245,246-7$, pl. I4

poppy 94, 97, II7, I21, I49, I76, I94, $2 \mathrm{I} I-\mathrm{I} 2$

corn I52, 23I, 244

field 99, I48, I89, 224

white 2 I I

wild 120

populeon (ointment) II4, I3I, 22I, $224^{-}-2,269$

pork fat, see animal products posset ale 87, I06, II7, I3I, I37, I49, I5I, 223-4, 233, 250, 258, 26 I

powder of worms, see worms pregnancy I5, 62, 97, I34, I34n., I56, I56n., I57, I57n., 200-1, 220, 222

prepared steel, see steel and steeled preparations

puerperal fever, see fever purslane 94, I48, I80, I95, 203, 2 I I

pustules, see skin disorders pyrethrum I03, II3, II5, I83, I96 quartan fever, see fever

quince I8o

see also oils

quinsy 208-I2

quotidian fever, see fever

radish 105, I7I, 220, 23I

raisins 84,93, II5, I26, I34, I42, I58-9, I65, I73, I85, 205, 207, 212, 254, 262, 280

realgar (arsenic) I29

red lead (oxide), see lead

respiratory complaints $2 \mathrm{I}-2,83-5$,

92-5, І I5-г6, І I8, І26-9, I32-3, I39-40, I65-6, I687I, I98-20I, 226-7, 255-7, $26 \mathrm{I}-3,277-82,289$

'retained products of conception', see placenta

rheum 77-8I, 86, 98, I24, I52, 295

rhodium wood 190

rhubarb i6, 79, 81, 85, 89, 92, 99-IOO, IO9, III, II4-I5, II8, I20-2I, I23, I30, I38-9, I48-9, I52-4, I58-9, г62-6, I73-4, I76, I82, I85-6, I90, 202, 207, 209, 213, 2I8-I9, 23I, 233, 240-2, 247, 250-I, 256, 260-I, 267, 269, 272, 274 , pl. I2

rock alum, see alum

rocket seed 98

rooster, see animal products

rose 89, 99, I09-IO, I20, I28, I76, I78, I9I, I97, I99, 203, 205, 2I $2,2 \mathrm{I} 4,280$

conserve $84,9 \mathrm{I}$, I08, I2I, I48, I52, I6I, 222, 268, 274

damask I70, I79, I90, I99

honey I36, I40, I44, I64, I80, I9I, 20I, 209, 2I2, 220

juice $85, \mathrm{I} 63,203,2 \mathrm{I} 3,227,240$, $24 \mathrm{I}$

julep I05, I08, 220

ointment 22I 


\section{Index of ailments and treatments}

powder $84,93,97$, IO3, IIO, I2I, I32, I7I-2, I76, I81, I87, I89, I94, I97, 20I, 24I, 268

sugar 92, І 16, І2 I, І23, І76-7, г86, 203, 2II, 229, 262

syrup 85, 92, I00, I09, I34, I36, I48, I6I-2, I77, I84, 230-I, $246,250,272$

tablets 94, II6, I2 I, I23, I76-7, 203 vinegar 220, 23I, 246 water 8I, 83, 86, 94, 98, Iо I, I Iо, I20, I24-5, I27, I36, I38, I44, I55-6, I64, I8I, I84, I94, 22I, 223, 225-6, 23I, 245, 267, 269 see also oils; sugar

rosemary 79, 90, I00, I03, I I4, I36, I40, I56, I58, I79, I84, I99, 207, 213, 223, 237, 267, 268, 280

rosewood 170

see also oils

roundworms 247, 266, 297 see also worms

ruby, see gems

rue 9o, I03, I04, IIо, II4, I27, I50,

see also oils

I55, 224, 233-5

rye $\mathrm{I} 54, \mathrm{I} 80,272$

safflower I22

saffron 8I, 85-6, IIo, II3, II5-I7, I2I, I23, I45, I63-4, I66, I8I, I99, 213, 219, 234, 237-8,

245, 247, 254, 26o, 262, 280, pl. 19

sage 90, II4, I28, I36, I40, I62, I67, $235,267,277$

see also oils

St John's wort I26, I86, 204

see also oils

salt 9o, 94, го5, І24, І37, І44, г67, I85, 220, 222, 23I

salt of tartar (potassium carbonate) 273

saltpetre I08, 247, 295 sandalwood 94, 24I

red I63, 2I3, 22I, 23I

white $163,2 \mathrm{I} 3$

yellow I I9, I32, I99, 243

sanicle 204

santalon 213, 242, 275

sapphire, see gems

sarcocol 8I, 83, I25

sarsaparilla I6, 80, 84, 86, 97, Іоо, III, II4, I36, I46-7, I53, I62, I66, I84-5, I90-I, 2I5-I6, 2I8, 24I, 26o, 264, 267, 269

sassafras 80, I00, III, II4, II9, I40, I53, I62, I66, I70, I79, I84, I90, I97, 218, 242, 260, 262, $264,267,274$

see also oils

scabious 84, 89-9I, 93, 97, 99, I I9, I20, I73, I76, I93, I94, 205, 2I2, 224, 23I, 243-4, 246, 259-6o, 264, 274, 281, pl. 4

scabs, see skin disorders

scammony I39, I86, 230

scarlet pimpernel I 27

scorbutic arthritis, see arthritis

scorbutic collapse, I78-9

see also scurvy

scorbutic dropsy I39-40, 258-66

see also scurvy

scorbutic epilepsy, I8I-3

see also scurvy

scorbutic fever, see fever

scorbutic weakness I92-3

see also scurvy

scorpion, see animal products; oils

scrotum I37, 2I4, 24I, 26I

see also urinary complaints

scurvy I5, 21, 31, 38-9, 39, 44-5, 48,

$59,62-5,70,78-9$, I $22-4$,

I67-8, I68-7I, I72-4, I84-5, I88-9, I90-I, I92-3, I93-4, I97, I98-201, 228-30, 232-4, $23^{6}-9,240,24^{8}-50,25^{\mathrm{I}}-7$, 259, 263-6, 266-7, 275-7, 278-8I, 295 


\section{Index of ailments and treatments}

scurvy (cont.)

see also scorbutic arthritis;

scorbutic collapse; scorbutic

dropsy; scorbutic epilepsy;

scorbutic weakness

scurvy-grass 21, 79-80, 99, I23-4, I40, I62, I68, I70-I, I73, I79,

I83-4, I89-9I, I94, I99, 201, 218, 233, 24I, 244, 255-6, 259-6I, 264-5, 276, pl. I I

sea-moss I95

sealed earth I07, I2I, I28, I77, I78,

272

sebesten (fruit) 98

self-heal I7o

seminal discharge $176-7$

senna I6, 6i, 79, 85, 87, 93, 98-іоo, I04, Iо8-9, II4-I5, I 18, I22, I25-6, I32, I36, I39, I4I, I46, I48, I52-4, I58-9, I62, I69, I73, I85, I90, I95, 207, 209, 216, 218, 235, 26I, 269, 274, pl. 7

sheep suet, see animal products silphium 274

sine quibus esse nolo (pills) I03, II8, I25, I26, I84, 247, 26I, 270, 277

skin disorders I5, 84, I37, 294

blotches 252

pimples 88

pustules I20, I36-7, I6I-2

rash I22, I36-7, 296

scabs 90-I, I5I, 275-7

spots $85-6$, I20, I22, I34, I43, I $67-8,232-4,237,246,295$

see also morphew

sleeping disorders I0, 22-4, Iо4-6,

I34, I39-40, I44, I56-7,

I66-7, I72-4, I8I-2, I87,

I90-I, 230-2, 252-5, 268-9,

$274,28 \mathrm{I}-2$

smallpox I5, I32-3, I43-5, 216-I9, $245^{-6}$

see also measles snails, see animal products

snakeweed I78, I95

sneezing powder I03, I06, I83, $25^{8}$

snowbells (snowdrops) I59

soapwort 84

sore throat I39-40, I4I-2, I64-5, 209-I2, 295

see also inflammation and swelling

sorrel I07, 203, 273

see also wood-sorrel

Spanish pellitory (pellitory of Spain), see pyrethrum

spasm of the mouth (tortura oris) 20, I I I-I3, I56, 239-4I, 257-8, 295

speedwell 84, 93, 274

spiders' webs, see animal products spikenard I00, I09, I23, I58, I63,

$$
\text { 20I, } 2 \mathrm{I} 3
$$

spitting up blood $270-2$

spleen 21, 63-4, 70, I39, I49-51, I5O, I63-4, I67-8, I73, I78-9,

I89, I93-4, 228-30, 233,

236-9, 238, 243, 248-9, 269,

277-81, 279, 295

spleenwort II9, I73, I99, 264, 274

splenic jaundice, see jaundice

spots, see skin disorders

spurge 202

squill (sea-onion) 85, 87, 89, 99, II5, II 9,243

see also oxymel of squills; vinegar

stag's heart bone, see animal

products

starch I25, I80, 2I I

steel and steeled preparations 2I, 79, 92, II0, II9, I23, I55, I6I, I7I, I79, I87, 213, 242, 265, 270

filings 2 I, I90, I99, 280

steeled milk I77

steeled water 88, 92, I80, 2I4, 229

steeled wine 2I, II9, I90, I94, I99-200, 233, 239, 24I, 257, $276,277,280$ 


\section{Index of ailments and treatments}

stomach conditions 9I IOI, I06, I33, I43, I47, I63-4, I72-4, I8I, 220, 234, 289, 292-3 295

abscess 89

ache 87, I53-4, I6I, 204-5, 208, 218, 268-70

pain $83-4$, I38, I98, 200-I, 245

wind $2 \mathrm{I}, 87-8$, $\mathrm{IO}^{-}-4$, I2 $\mathrm{I}-2$, I32-3, I49-5I, I53-4, I74-6, I87, I98-20I, 236-9, 248-50, $255^{-7}, 26 \mathrm{I}-7,288,297$

see also abdominal pain; pain in the side; inflammation and swelling

stone $\mathrm{I} 88^{-}-9,237^{-8}, 25^{-}-\mathrm{I}, 295^{-6}$

see also kidneys

strawberry 99, Io9

styrax 84, II4, II5, I29, I66, I67, I8I, 20I, $24 \mathrm{I}$

'suffocation of the mother', see uterine conditions

'suffocation of the womb', see uterine conditions

sugar 62, 79, 8I, 85, 90, 92, 94, 98, IOI, IO4, IO7, IO9-IO, II4, II9, I2I, I26, I3I-2, I4I, I63, I69, I76, I79, I84-7, I95-7, 202, 2I5, 2I8-I9, 22I, 223, 227, 230, 233, 235, 237, 247, 250, 262, 264-5, 267, 269, 277

'best' I53, I99, 267

brown 92, I80, 272

'candy' 81, 84, 88, II5-16, I34, I $54,203,205,254^{-5}$

lavender 207

red I40, I5I

rose 92, 94, Ііо, Іі6, І2 I, І23-4, I76-7, I8I, I86, 203, 21 I, 229, 262, 267

unrefined Io8, 236, 247, 249, 259

violet $98, \mathrm{I} 39,246$

white 94, I04, I38, I97, 21 I, 216, 263

see also barley sugar sulphur $84, \mathrm{I} 37, \mathrm{I} 77,265,280$

balsam 226

flowers of 85, I08, I18, I29, I98, 217,226

'live' I24, I85

rock 90

see also oils

sulphuric acid I99

sweet flag 80, 93, I04, II4-I5, I I9, I32, I58, I70, I9o, I96, I99, 207, 209, 242-3

swallows' nest, see animal products swelling, see inflammation 'syrup of the five roots' I3o

tacamahacca powder I70 tamarind I76, I85, I93-4, I98, 213, $227,263,270$

tamarisk I09, II9, I22-3, I9o, I99, 264,267

tapsi valentia 225

tarragon 145

tartar 20I, 250, 274, 275, 280

crystal 90, I73, I9I, 238, 274, 278

vitriolated $\mathrm{I} 68,278$

see also cream of tartar; salt of tartar; oils

tenesmus 88, I80-I, 296

see also bowel disorders

tertian fever, see fever

testicles I3O, I49-5 I

thirst 98-9, I08, I34, I43, I45,

I49-52, I68-7I, I84, 205,

248-5I, 258, 262, 275, 28I,

290

throat conditions, see inflammation and swelling; sore throat

tinnitus $269-70$

see also auditory ailments

tiredness 2I, I98-200, 263-6, 275,

$277^{-8}$

tobacco Io6, I98

tongue $\mathrm{I} 44, \mathrm{I} 64-5, \mathrm{I} 67-8,220-2$,

244,249

see also inflammation and swelling 


\section{Index of ailments and treatments}

tonsils I39-43, 2I I

see also inflammation and swelling

toothache 6I, I35-6, I48-9, I56-7, I9I, 226, 237-8, 240-I

tormentil I28, I9I, I95, 204

tragacanth гі6, г27, I46, г77, І80, I86, 202, 2II, 2I4-I6

trembling and tremor IOI, IO9-IO, II6-I7, I8I-2, I93-4, 223, 263-6

see also palpitations

turbith I32, I39, I86, 265

turmeric 85

turpentine 81, 84, 90, I07, II5, I25, I28-9, I46, I80, I86, 202-3, 2I $4-\mathrm{I} 5,226$

Cyprus 201, 229, 250, 256, 267

Venice I28

see also oils

tutty (zinc oxide) 8I, 86, I I I, I25, 2 I 4

tympany I96-7, 296

see also dropsy

ulceration and ulcers $77-8, \mathrm{I} 5 \mathrm{I}-2$, I80, 2I4-I6, 215, 275-7, 289, 290, 293, 296

see also canker

umbilicus II7-I8, I31, I58-9, I84, 228-30

'Ungaric' fever, see fever urethra 2I4-I6, 215

see also urinary complaints

urinary complaints I6, 64, 67-70, 80, I02, I37, I38, I4I, I43, I 4 6-7, I88-9, 208, 2 I4-I6, $246-7,290$, pl. 25

uterine conditions

'affection of the womb' i $8-$-19

'fit of the mother' 7I, I97, 234-5, 237-8, 244, 257-8, 266-7, $272-5$

'fit of the womb', see 'fit of the mother'

'motion of the womb' 70, I95 'suffocation of the mother' 70, I3I, I57, I94, I95, 2 I6-I8, $244^{-5}, 257^{-8}, 293,296$ 'suffocation of the womb', see 'suffocation of the mother' uterine flux 98-9, 29I 'uterine pains' 228-30 'uterine passion' 2I6-I8 uterine wind $192-3,296$ see also flux

uvula I4I-2, 208-I2

see also throat conditions

valerian I27, 264

veal, see animal products

veins 6o, 9I, I05, I06, I65, 220, 288-9, 29I, 293-4

basilic 162

brachial 243, 273

cephalic 98, I03, I57

haemorrhoidal 6o, I06, I24, 207, $24 \mathrm{I}-2,273,292$

hepatic 6o, 24I

jugular 209

kidneys 204

liver 292-3

lung I2 I, 270

meseraic 63-4, 70, 9I, 248-9, 292

see also venesection

venesection (phlebotomy) 43, 6o, 66, 87, 90, 105, I06, Іо8, І20, I30, I57, I62, I64-5, 209, 2II, 2I3, 220, 23I , 232, 24I, 26 I

Venice treacle 6i, IIо, II I, I 7 , I63, I7I-2, I77, I83-4, I89, 2I3, 242

Venice turpentine, see turpentine verdigris I24

vetch 170

vinegar 83, 86-7, 93, I05, I Iо, II9-20, I23-4, I35, I37, I54, I64, I95, 203, 2I7, 274

red 83 


\section{Index of ailments and treatments}

rose 220,23 I, 246

squills 89, I I9, 243

wine $192,24 \mathrm{I}$

violets $87,89,94-5,98$-гоо, го4, Io6-7, I09, I34, I39, I49, I52, I62-3, I65-6, I77, 203, 205, 222, 23I, 240, 246, 256, 265,272

see also oils

vitriol I57, 222, 274

spirit of I05, I9I, 220

white I25, I5I, I56

see also oils; tartar

vitriolated tartar, see tartar

vomiting 72, 97-IOI, II $5^{-1} 6$, I3 $3^{\mathrm{I}-2}$, I $43-4$, I47-8, I62, I68-7I, I93-4, 200-I, 222, 255-7, 268-9, 275-7

vomiting blood I2 I, I62-3

wakefulness, see sleeping disorders

wallflower 158

walnut (tree) 224

see also nuts

wandering gout, see gout

water of mercury, see aquae (waters)

waters of St Vincent's Spring 216, 266

water-lilies 94, 203

see also oils

water-melon 2 I I

watercress $2 \mathrm{I}, 79^{-80}, 9^{0}, \mathrm{I} 58, \mathrm{I} 62$, I68, I70-I, I73, I79, I84, I99, 200, 218, 233, 237-8, 244, 256, 259-6o, 26i, 264, 276

wax, see animal products

wheat barley, see barley

wheat I66, I70, 2 I9, 246, 297

see also bread and breadcrumbs

white dittany, see dittany

white lead, see lead

white vitriol, see vitriol

wind, see stomach conditions wine IOI, II9, I27, I32- 3, I54-5, I60, I69, I77, I9I, I95, 200, 203-4, 262, 280

claret I44, I50, I55

malmsey 88, 97, I04

muscadel 85

red I07, I26, I80

Spanish 93, I50, I6 I

spirit 94, 229

white 79, 81, 86, 89, го4, І І6, I22-3, I26, I30, I37-8, I55-7, I63-4, I90, I96, I99-200, $213,265,274$

see also steel and steeled preparations

winter cherry (nightshade) I37, I86, 202, 2I $5^{-1} 6,243,264,267$

wintergreen 204

wood-sorrel 240, $25^{8}$

woodbine 209

womb, see uterine conditions

worms $6 \mathrm{I}-2$, 92, II7-I8, I3 ${ }^{\mathrm{I}-2}$,

I47-9, I58-9, I83-4, I95,

222, 230, 247, 266, 297

see also roundworms

worms, powder of I69, I82, I84

see also earthworms; oils

wormseed 62, 247

wormwood 79, 87, 89-9I, I00, I04, II8-I9, I22-3, I32, I42, I59, I6I, I64, I68-7I, I73, I90, I95, 2I I , 2I 8, 224, 233, 24I, $255,260,264,274,277 \cdot$ pl. I3

French 195

see also oils

yarrow I26, I55

yeast (brewer's) 80, 264-5

flowers of 153, I 70

yellow choler, see humours

yellow jaundice, see jaundice

zedoary I54, I96, 262

zircon II7 